\title{
Different types of atrial fibrillation, renal function, and mortality in elderly Chinese patients with coronary artery disease
}

This article was published in the following Dove Press journal:

Clinical Interventions in Aging

14 February 2014

Number of times this article has been viewed

\author{
Shihui Fu',* \\ Tao Liu, ${ }^{2, *}$ \\ Leiming Luo' \\ Ping $\mathrm{Ye}^{\prime}$ \\ 'Department of Geriatric Cardiology; \\ ${ }^{2}$ Nanlou Ultrasound Department, \\ Chinese People's Liberation Army \\ General Hospital, Beijing, People's \\ Republic of China
}

*These authors are joint first authors

Correspondence: Leiming Luo Department of Geriatric Cardiology, Chinese People's Liberation Army General Hospital, 28 Fuxing Road, Beijing 100853, People's Republic of China

Tel +86 I0 88626362

Fax +861066876349

Email lleim@sina.com
Background: Atrial fibrillation (AF) is the most common arrhythmia in patients with chronic kidney disease (CKD), and the combined prevalence of these two disorders increases as the population ages. Both AF and CKD have risk factors for development of each other and eventual mortality. However, the relationship between different types of $\mathrm{AF}, \mathrm{CKD}$, and mortality remains unclear, especially in elderly Chinese patients with coronary artery disease.

Methods: This study comprised 1,050 patients of median age 86 (60-104) years with coronary artery disease. The end point was all-cause death during a mean follow-up of 417 days.

Results: Of 219 patients identified to have AF, 128 had paroxysmal type, 44 had persistent type, and 47 had permanent type. After adjusting for confounders, the estimated glomerular filtration rate was lower and the prevalence of CKD was higher in patients with permanent AF but not in those with paroxysmal or persistent AF. During follow-up, 106 non-CKD patients and 112 CKD patients died; mortality was significantly higher in CKD patients with AF than in those without $\mathrm{AF}$ (36 [40.9\%] versus 76 [26.8\%]), but not in patients without CKD (17 [13.0\%] versus 89 [16.3\%]). In patients with CKD, paroxysmal AF was independently associated with higher mortality after adjustment but not persistent or permanent AF. No type of AF had an independent association with mortality in patients without CKD.

Conclusion: All types of AF had a high prevalence. Permanent AF was independently associated with an increased prevalence of CKD and a decreased estimated glomerular filtration rate. Paroxysmal AF was an independent risk factor for survival in patients with CKD but not in those without CKD.

Keywords: atrial fibrillation, chronic kidney disease, coronary artery disease, Chinese, elderly, mortality

\section{Introduction}

Atrial fibrillation (AF) is a cardiac arrhythmia seen frequently in clinical practice and increases in prevalence with advancing age. ${ }^{1-5} \mathrm{AF}$ is also the most common rhythm abnormality in patients with chronic kidney disease (CKD). ${ }^{6}$ An estimated 19\%-24\% of patients with CKD are diagnosed with AF, and the combined prevalence of these conditions increases as the population ages. AF and CKD share a number of risk factors, including increasing age and pre-existing cardiovascular disease, including hypertension, diabetes mellitus, coronary artery disease (CAD), and chronic heart failure, and the close relationship between AF and CKD has attracted attention. ${ }^{7-9}$ Both AF and CKD confer a significant morbidity burden and are associated with a worse outcome. ${ }^{10-12}$ With increasing life expectancy, the elderly are the most rapidly expanding portion 
of the world population, making the association between AF, CKD, and mortality an even more significant public health concern.

The close link between AF and renal function has been shown in previous studies involving patients with endstage renal disease (ESRD). ${ }^{13-16}$ However, patients with ESRD represent a small proportion of individuals with decreased renal function, and much less is clear about the correlation between AF and less severe decline in renal function. ${ }^{17-21}$ Whether different types of AF have different associations with renal function also remains unclear, and may have potential clinical implications for the management of high-risk patients. Meanwhile, age, race, and comorbidity have an unavoidable effect on the bidirectional relationship between AF and renal function, and taking these factors into consideration is necessary for an indepth analysis of this relationship. However, few studies have addressed this association in elderly Chinese patients with CAD.

A close relationship has been demonstrated between AF and poor clinical outcome in patients with ESRD., ${ }^{2,22}$ However, whether the relationship is similarly present in patients with less severe decline of renal function has not been extensively studied. ${ }^{23}$ Different types of AF may have different prognostic significance, and further studies are needed to elucidate this possibility. Given the contribution of age, race, and comorbidity to the complexity of the problem, we felt a study was necessary to evaluate the impact of different types of AF on mortality in elderly Chinese CAD patients with and without CKD. To address the knowledge gap, the current study investigated the prevalence of different types of AF in elderly Chinese patients with CAD, assessed the burden of decreased renal function on development of different types of AF, and evaluated the influence of different types of AF on survival in patients stratified according to the presence of CKD.

\section{Methods}

\section{Study population}

The study cohort comprised 1,050 Chinese patients aged $\geq 60$ years with CAD who were enrolled consecutively at the Department of Geriatric Cardiology, Chinese People's Liberation Army General Hospital. Their median age was 86 (range 60-104) years. The Chinese People's Liberation Army General Hospital was their designated hospital and held their integrated long-term medical and final death records, which made it easier for us to follow them up effectively and judge endpoints accurately. Using the American College of Cardiology (ACC)/American Heart Association (AHA) or European Society of Cardiology (ESC) guidelines, the lead physicians identified patients with CAD on the basis of history, symptoms of typical angina, cardiac markers, and specific cardiac examinations, including electrocardiogram (resting and exercise), echocardiography, radionuclide imaging, computed tomography, and coronary angiography. ${ }^{24-26}$

\section{Definition of study variables}

Body mass index was calculated as weight in kilograms divided by the square of height in meters. Mean systolic and diastolic blood pressure (BP) was systematically determined from five measurements. A standard 12-lead electrocardiogram was recorded in all participants using strictly standardized procedures with identical electrocardiographic equipment. Several structural parameters were derived from the echocardiographic data. Left ventricular ejection fraction was measured by Simpson's method. ${ }^{27}$ Left ventricular mass was calculated according to the formula described by Devereux et al, ${ }^{28}$ and left ventricular mass index was obtained by dividing the left ventricular mass by body surface area. In light of the ACC/AHA/ESC guidelines, patients with $\mathrm{AF}$ were detected by the lead physicians on the basis of medical record documentation and electrocardiographic evidence, and classified as paroxysmal, persistent, or permanent. ${ }^{29}$ Chronic heart failure was diagnosed by the lead physicians according to the ESC guidelines. ${ }^{30}$ Hypertension was defined as either mean systolic $\mathrm{BP} \geq 140 \mathrm{mmHg}$, mean diastolic BP $\geq 90 \mathrm{mmHg}$, or use of antihypertensive medication. Diabetes mellitus was defined as being present if participants had a fasting serum glucose $\geq 7 \mathrm{mmol} / \mathrm{L}$ or used insulin or oral hypoglycemic medications. History of smoking was defined as a history of cigarette use on a regular basis (more than one cigarette per day). Estimated glomerular filtration rate (eGFR) was calculated using the modified Modification of Diet in Renal Disease equation based on data for the Chinese population:

$$
\begin{gathered}
175 \times \text { serum creatinine }(\mathrm{mg} / \mathrm{dL})^{-1.234} \times \text { age }(\text { years })^{-0.179} \\
(\times 0.79 \text { if female }) .^{31}
\end{gathered}
$$

CKD was defined as eGFR $<60 \mathrm{~mL} /$ minute $/ 1.73 \mathrm{~m}^{2}$ according to the recommendations of the National Kidney Foundation. ${ }^{32}$ Other blood assays, including hemoglobin, serum albumin, glucose, triglycerides, high-density lipoprotein cholesterol, low-density lipoprotein cholesterol, and serum sodium, potassium, calcium and phosphorus were carried out at the central laboratory in the Department of Biochemistry, Chinese People's Liberation Army General Hospital. 


\section{Follow-up and outcome}

Given the high incidence of multiple organ failure in the elderly as well as the priority of all-cause mortality in outcome studies, the primary end point assessed in the current study was all-cause death. The mean follow-up period consisted of 417 days (median 317 days, interquartile range 185-557 days). No patient dropped out during the study period. Follow-up data were tracked directly from medical records and telephone interviews. Death was ascertained from the death record, ie, a legal document including time, site, and other information.

\section{Statistical analysis}

Patient data were described by the mean and standard deviation for continuous variables with a normal distribution, median and interquartile range for non-normally distributed continuous variables, and number and percentage of the total for categorical variables. Characteristics between patients with different types of AF and those without AF were matched using the Student's $t$-test for continuous variables (normal distribution), the MannWhitney $U$ test for continuous variables (abnormal distribution), and the chi-squared test for categorical variables. Multinomial logistic regression analysis was used to observe the relationship between eGFR and CKD with different types of AF after adjusting for age, sex, history of smoking, body mass index, chronic heart failure, hypertension, diabetes mellitus, mean systolic and diastolic BP, heart rate, left ventricular ejection fraction, interventricular septum, left ventricular posterior wall, left ventricular end-systolic diameter, left ventricular enddiastolic diameter, left ventricular mass index, hemoglobin, serum albumin, glucose, triglycerides, high-density lipoprotein cholesterol, low-density lipoprotein cholesterol, and serum sodium, potassium, calcium, and phosphorus. Cox regression models including adjustment for the same confounders as described above were used to examine the adjusted effect of different types of AF on mortality for the patients grouped by presence of CKD. A two-tailed $P<0.05$ was considered to be statistically significant. All analyses were done using Statistical Package for the Social Sciences version 17 software (SPSS Inc, Chicago, IL, USA).

\section{Results}

The study cohort comprised 1,050 patients, of whom $219(20.9 \%)$ had a diagnosis of AF. Among the patients with AF, 128 (12.2\%) had paroxysmal AF, 44 (4.2\%) had persistent $\mathrm{AF}$, and $47(4.5 \%)$ had permanent AF.
Demographic and clinical characteristics according to presence and types of AF are summarized in Table 1. Compared with non-AF patient group, the group of patients with permanent AF $(P<0.05)$, but not those with paroxysmal AF $(P>0.05)$ or persistent AF $(P>0.05)$, contained a significantly higher proportion of patients with CKD. Compared with non-AF patients, eGFR was lower in patients with permanent AF $(P<0.05)$ but not in those with paroxysmal AF $(P>0.05)$ or persistent AF $(P>0.05)$. Meanwhile, chronic heart failure was significantly more common in patients with any type of AF than in non-AF patients (all $P<0.05$ ). In addition, there were several differences in echocardiographic, blood pressure, and electrolyte parameters between non-AF patients and those with any type of AF (all $P \leq 0.05$ ).

Table 2 shows the factors associated with the different types of AF after adjusting for age, sex, history of smoking, body mass index, chronic heart failure, hypertension, diabetes mellitus, mean systolic and diastolic BP, heart rate, left ventricular ejection fraction, interventricular septum, left ventricular posterior wall, left ventricular end-systolic diameter, left ventricular end-diastolic diameter, left ventricular mass index, hemoglobin, serum albumin, glucose, triglycerides, high-density and low-density lipoprotein cholesterol, and serum sodium, potassium, calcium, and phosphorus. eGFR was independently lower and the prevalence of CKD was independently higher in patients with permanent $\mathrm{AF}$ (all $P \leq 0.05$ ) but not in those with paroxysmal AF (all $P>0.05$ ) or persistent $\mathrm{AF}$ (all $P>0.05$ ).

During follow-up, 106 (15.6\%) non-CKD patients and $112(30.1 \%)$ CKD patients died; mortality was significantly higher in AF patients with CKD than in non-AF patients with CKD (36 [40.9\%] versus 76 [26.8\%], respectively, $P<0.05)$ but not in those without CKD $(17$ [13.0\%] versus 89 [16.3\%], respectively, $P>0.05$ ). Table 3 shows the factors correlated with the increased risk of mortality in CKD patients in comparison with non-CKD patients after adjustment for the same confounders as described above. In patients with $\mathrm{CKD}$, paroxysmal $\mathrm{AF}$ was strongly associated with a higher independent risk of death $(P<0.05)$, while persistent $\mathrm{AF}$ and permanent $\mathrm{AF}$ were not associated with a higher independent risk of death (all $P>0.05$ ). In contrast, any type of AF had no independent association with mortality (all $P>0.05$ ) in patients without CKD. Further, regardless of whether or not patients had CKD, serum levels of hemoglobin, albumin, triglycerides, and phosphorus, as well as heart rate, consistently showed an independent effect with regard to survival. 


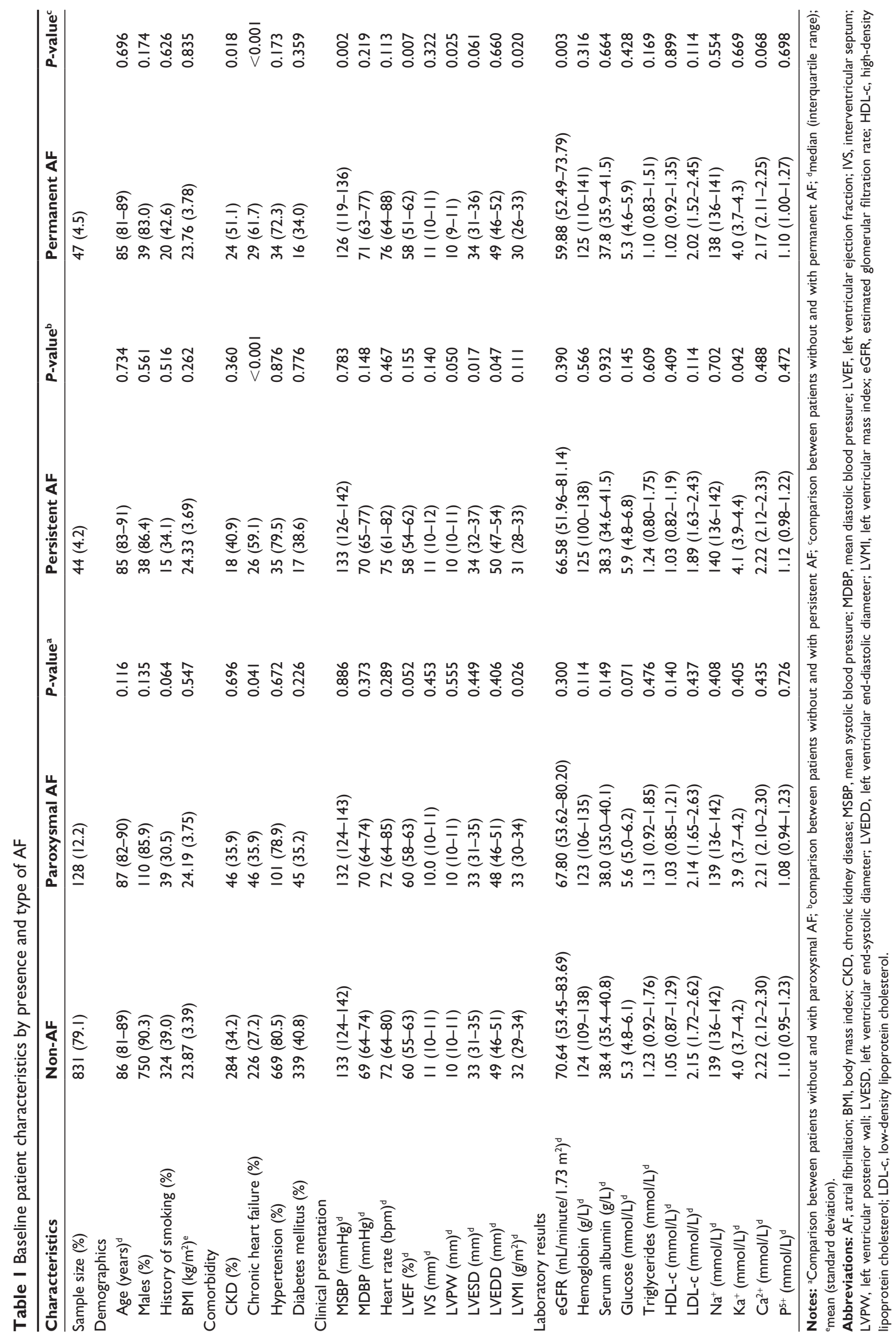


Table 2 Multinomial logistic regression model results for different types of AF

\begin{tabular}{|c|c|c|c|c|c|c|}
\hline \multirow{2}{*}{$\begin{array}{l}\text { Different models } \\
\text { Dependent } \\
\text { variable }\end{array}$} & \multicolumn{3}{|c|}{ Model with eGFR as a variable ${ }^{a}$} & \multicolumn{3}{|c|}{ Model with CKD as a variable ${ }^{a}$} \\
\hline & $\begin{array}{l}\text { Independent } \\
\text { variables }\end{array}$ & HR (95\% Cl) & $P$-value & $\begin{array}{l}\text { Independent } \\
\text { variables }\end{array}$ & HR (95\% Cl) & $P$-value \\
\hline \multirow[t]{5}{*}{ Paroxysmal AF } & eGFR (mL/minute/l.73 m²) & $0.999(0.990-1.008)$ & 0.824 & CKD & $0.909(0.583-1.418)$ & 0.675 \\
\hline & Age (years) & $1.032(0.999-1.066)$ & 0.055 & Age (years) & $1.033(1.000-1.067)$ & 0.049 \\
\hline & $\mathrm{CHF}$ & $1.926(1.214-3.053)$ & 0.005 & $\mathrm{CHF}$ & $1.956(1.235-3.099)$ & 0.004 \\
\hline & Heart rate (bpm) & $1.014(1.001-1.026)$ & 0.033 & Heart rate (bpm) & $1.013(1.001-1.026)$ & 0.035 \\
\hline & HDL-c (mmol/L) & $0.454(0.218-0.945)$ & 0.035 & HDL-c (mmol/L) & $0.446(0.214-0.928)$ & 0.031 \\
\hline \multirow[t]{4}{*}{ Persistent AF } & eGFR (mL/minute/l.73 m²) & $1.006(0.99 \mid-1.022)$ & 0.424 & CKD & $0.805(0.385-\mid .68 I)$ & 0.564 \\
\hline & Age (years) & $1.053(0.999-1.1 \mathrm{II})$ & 0.055 & Age (years) & $1.052(0.998-1.110)$ & 0.058 \\
\hline & $\mathrm{CHF}$ & 4.577 (2.189-9.567) & $<0.001$ & $\mathrm{CHF}$ & $4.529(2.172-9.446)$ & $<0.001$ \\
\hline & MDBP $(\mathrm{mmHg})$ & $1.055(1.005-1.108)$ & 0.032 & MDBP $(\mathrm{mmHg})$ & $1.055(1.005-1.108)$ & 0.030 \\
\hline \multirow[t]{7}{*}{ Permanent AF } & eGFR (mL/minute/l.73 m²) & $0.975(0.958-0.993)$ & 0.007 & CKD & $2.005(1.002-4.014)$ & 0.050 \\
\hline & Age (years) & $1.069(1.012-1.129)$ & 0.018 & Age (years) & $1.072(1.015-1.133)$ & 0.012 \\
\hline & $\mathrm{CHF}$ & $5.082(2.436-10.605)$ & $<0.001$ & $\mathrm{CHF}$ & $5.044(2.415-10.538)$ & $<0.001$ \\
\hline & MSBP (mmHg) & $0.936(0.902-0.97 I)$ & $<0.001$ & MSBP (mmHg) & $0.938(0.904-0.973)$ & 0.001 \\
\hline & MDBP $(\mathrm{mmHg})$ & $1.110(1.05 I-1.172)$ & $<0.001$ & MDBP $(\mathrm{mmHg})$ & I.I07 (1.049-I.169) & $<0.001$ \\
\hline & Hemoglobin $(g / L)$ & 1.021 (I.00I-I.042) & 0.041 & Hemoglobin $(g / L)$ & $1.019(0.999-1.040)$ & 0.063 \\
\hline & Serum albumin $(g / L)$ & I.I0I (0.997-I.216) & 0.058 & Serum albumin $(g / L)$ & $1.096(0.992-1.210)$ & $0.07 I$ \\
\hline
\end{tabular}

Notes: adjusted for age, sex, history of smoking, body mass index, chronic heart failure, hypertension, diabetes mellitus, mean systolic blood pressure, mean diastolic blood pressure, heart rate, left ventricular ejection fraction, interventricular septum, left ventricular posterior wall, left ventricular end-systolic diameter, left ventricular end-diastolic diameter, left ventricular mass index, hemoglobin, serum albumin, glucose, triglyceride, high density lipoprotein cholesterol, low density lipoprotein cholesterol, serum sodium, potassium, calcium and phosphorus.

Abbreviations: AF, atrial fibrillation; bpm, beats per minute; HR, hazard ratio; Cl, confidence interval; eGFR, estimated glomerular filtration rate; CKD, chronic kidney disease; CHF, chronic heart failure; HDL-c, high-density lipoprotein cholesterol; MDBP, mean diastolic blood pressure; MSBP, mean systolic blood pressure.

\section{Discussion}

The current study shows that regardless of type, there is a high prevalence of AF in elderly Chinese patients with CAD. Moreover, the study confirms that patients with permanent AF have a lower eGFR and a higher prevalence of CKD, even after adjusting for a broad set of potential confounders. Further, it is demonstrated that, after full adjustment, paroxysmal AF is a strong predictor of an independently increased mortality rate in patients with CKD relative to those without CKD. These observations are extremely relevant to the care of this elderly population and deserve close attention.

In previous studies of patients with ESRD, the prevalence of AF ranged from $3.5 \%$ to $27 \%$, depending on the patterns of AF (paroxysmal, persistent, permanent) and associated risk factors. ${ }^{16}$ In the general hemodialysis population, the prevalence of AF was $27.0 \%$, however when patients with paroxysmal AF were excluded, the prevalence was $23.5 \%$, and when only those with permanent AF were included, the prevalence was $13.9 \% .{ }^{16}$ Patients with ESRD, however, represent a small proportion of individuals with a decreased renal function, and the correlation of AF with less severe decline of renal function has not been well evaluated. ${ }^{17-21} \mathrm{In}$ the Atherosclerosis Risk in Community study, Alonso et al proposed a progressively higher risk of AF with CKD. ${ }^{17}$ Horio et al conducted a study in 1,118 hypertensive patients and revealed an increased risk of AF in those with CKD. ${ }^{21}$ In contrast, a study of patients with CKD found that the association between eGFR level and AF prevalence was no longer significant after adjustment. ${ }^{20}$ Moreover, the association between different types of AF and renal function may have implications for the clinical management of high-risk patients. Meanwhile, age, race, and comorbidity had an unavoidable effect on the relationship between AF and renal function, and taking these factors into consideration is important for indepth analysis of this relationship. However, data on the association between different types of $\mathrm{AF}$ and renal function is extremely limited in elderly Chinese patients with CAD. The observations from our study further confirm that the relationship between renal function and permanent $\mathrm{AF}$ is independently significant, which is an important supplement to previous reports ${ }^{17-21}$ and subdivides this association in elderly Chinese patients with CAD.

Multiple pathophysiological mechanisms could underlie the relationship between AF and renal function. Neurohormonal activation has been implicated in the progression of renal damage and development of cardiovascular disease. Activation of the renin-angiotensin-aldosterone system has been documented in patients with CKD, and there is evidence suggesting that angiotensin also induces cardiac fibroblast proliferation and cardiac hypertrophy. ${ }^{33,34}$ Meanwhile, increased sympathetic activity has been proposed 
Table 3 Cox regression model results for survival of patients with and without CKD

\begin{tabular}{|c|c|c|c|}
\hline Population & $\begin{array}{l}\text { Independent } \\
\text { variables }^{\mathrm{a}}\end{array}$ & HR (95\% Cl) & $P$-value \\
\hline Without & Paroxysmal AF & $0.726(0.364-1.449)$ & 0.364 \\
\hline \multirow[t]{7}{*}{ CKD } & Persistent AF & $0.712(0.20 \mathrm{I}-2.527)$ & 0.600 \\
\hline & Permanent AF & $0.597(0.143-2.49 \mid)$ & 0.479 \\
\hline & Heart rate (bpm) & $1.017(1.006-1.029)$ & 0.003 \\
\hline & Hemoglobin $(g / L)$ & $0.975(0.963-0.987)$ & $<0.001$ \\
\hline & Serum albumin $(g / L)$ & $0.936(0.878-0.997)$ & 0.042 \\
\hline & Triglycerides $(\mathrm{mmol} / \mathrm{L})$ & $1.274(1.028-1.578)$ & 0.027 \\
\hline & $\mathrm{P}^{5+}(\mathrm{mmol} / \mathrm{L})$ & $2.211(1.233-3.966)$ & 0.008 \\
\hline With & Paroxysmal AF & $1.972(1.103-3.527)$ & 0.022 \\
\hline \multirow[t]{8}{*}{ CKD } & Persistent AF & $1.326(0.57 \mid-3.076)$ & 0.511 \\
\hline & Permanent AF & $0.506(1.180-1.425)$ & 0.197 \\
\hline & Age (years) & 1.051 (1.014-1.089) & 0.006 \\
\hline & Heart rate (bpm) & $1.015(1.001-1.030)$ & 0.038 \\
\hline & Hemoglobin $(g / L)$ & $0.984(0.973-0.996)$ & 0.007 \\
\hline & Serum albumin $(g / L)$ & $0.889(0.839-0.942)$ & $<0.001$ \\
\hline & Triglycerides $(\mathrm{mmol} / \mathrm{L})$ & $0.743(0.562-0.983)$ & 0.038 \\
\hline & $\mathrm{P}^{5+}(\mathrm{mmol} / \mathrm{L})$ & $2.302(1.545-3.429)$ & $<0.001$ \\
\hline
\end{tabular}

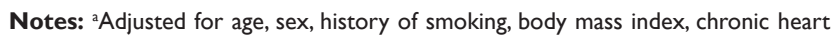
failure, hypertension, diabetes mellitus, mean systolic blood pressure, mean diastolic blood pressure, heart rate, left ventricular ejection fraction, interventricular septum, left ventricular posterior wall, left ventricular end-systolic diameter, left ventricular end-diastolic diameter, left ventricular mass index, hemoglobin, serum albumin, glucose, triglycerides, high density lipoprotein cholesterol, low density lipoprotein cholesterol, serum sodium, potassium, calcium and phosphorus.

Abbreviations: $\mathrm{CKD}$, chronic kidney disease; $\mathrm{HR}$, hazard ratio; $\mathrm{Cl}$, confidence interval; $A F$, atrial fibrillation.

to promote arrhythmogenesis in patients with $\mathrm{CKD} \cdot{ }^{33} \mathrm{In}$ addition to neurohormonal activation, inflammation has been considered as a contributor to the increased risk of AF in patients with CKD. Inflammatory markers are elevated in patients with CKD, and inflammation plays a significant role in the pathogenesis of AF. ${ }^{19}$ Further, structural and electrical atrial remodeling has shed light on the epidemiology of AF in patients with CKD. Prior studies have noted that increasing left atrial diameter and left ventricular hypertrophy related to $\mathrm{CKD}$ contributes to an increased risk of AF. ${ }^{19}$

A strong association between AF and mortality has been found in patients with ESRD.,22 For example, a study by Genovesi et al in 476 patients from five dialysis centers in one region of Italy reported that patients with AF had a $65 \%$ higher relative risk of death than those without $\mathrm{AF}^{22}$ Beyond that, in the Framingham study, Kannel et al found that AF was associated with a 2-3-fold increased risk of mortality in patients with ESRD. ${ }^{2}$ However, few studies have analyzed the association between AF and mortality in patients with a less severe decline in renal function. ${ }^{23}$ Nakagawa et al published data on 387 Japanese patients, reporting increased mortality rates in patients with $\mathrm{AF}$ and decreased eGFR. ${ }^{23}$
Considering that age, race, and comorbidity complicate matters further and that different types of AF may have diverse prognostic significance, the present study provides compelling data indicating that elderly Chinese AF patients with $\mathrm{CKD}$, but not those without $\mathrm{CKD}$, have an independently increased risk of mortality. Moreover, the present study findings confirm that the combination of CKD and paroxysmal AF has an independent effect on mortality rate, extending the results of previous studies and detailing the relationship between AF, CKD and mortality in elderly Chinese patients with CAD in more depth.

Several hypotheses have been offered to explain these findings. One possible explanation for the higher mortality rate is a higher incidence of stroke in patients with CKD and AF. It is well established that AF is one of the strongest predictors of ischemic stroke. ${ }^{35}$ A paper reported by Hart et al supports a higher rate of ischemic stroke in patients with $\mathrm{CKD}$ and $\mathrm{AF}$ in the Stroke Prevention in Atrial Fibrillation trials. ${ }^{36} \mathrm{~A}$ more plausible explanation linking the increased mortality rate with $\mathrm{CKD}$ and $\mathrm{AF}$ is that the presence of $\mathrm{AF}$ reflects the composite effect of a spectrum of cardiovascular disease mechanisms leading to left atrial enlargement, including vascular stiffness/noncompliance (due to classic atherosclerosis and nonclassic risk factors) present in the metabolic milieu of CKD (ie, abnormal calcium and phosphate metabolism, left ventricular hypertrophy, and sympathetic overactivity). ${ }^{37}$ The genesis of AF in patients with CKD is intimately linked with the same mechanisms that predispose these patients to increased risk of cardiovascular death. ${ }^{37}$

In conclusion, the present study finds that all types of $\mathrm{AF}$ have a high prevalence in elderly Chinese patients with CAD. Moreover, it underscores that patients with permanent AF are at extremely high risk of increased CKD and decreased eGFR independent of a broad set of potential confounders. Further, after adjustment for these potential confounders, the importance of paroxysmal AF as a strong risk factor for mortality in patients with CKD, but not in those without CKD, is emphasized. It is generally accepted that both CKD and AF are prevalent in the elderly, and thus our findings may have significant public health and clinical implications. Future studies should further delineate the potentially modifiable pathways responsible for this association.

\section{Acknowledgments}

This study was supported by grants from the National Key Basic Research Project (2012CB517503, 2013CB530804), 
the Health Special Scientific Research Subject of the Chinese People's Liberation Army (12BLZ34), and the Clinical Scientific Research Project of the Chinese People's Liberation Army General Hospital (2012FC-TSYS1021).

\section{Disclosure}

The authors report no conflict of interest in this work.

\section{References}

1. Go AS, Hylek EM, Phillips KA, et al. Prevalence of diagnosed atrial fibrillation in adults: national implications for rhythm management and stroke prevention: the Anticoagulation and Risk Factors in Atrial Fibrillation (ATRIA) Study. JAMA. 2001;285:2370-2375.

2. Kannel WB, Abbott RD, Savage DD, et al. Epidemiologic features of chronic atrial fibrillation: the Framingham study. $N$ Engl J Med. 1982;306:1018-1022.

3. Feinberg WM, Blackshear JL, Laupacis A, et al. Prevalence, age distribution, and gender of patients with atrial fibrillation. Analysis and implications. Arch Intern Med. 1995;155:469-473.

4. Kistler PM, Sanders P, Fynn SP, et al. Electrophysiologic and electroanatomic changes in the human atrium associated with age. $J$ Am Coll Cardiol. 2004;44:109-116.

5. Kamanth S, Lip GY. Atrial fibrillation in the elderly: anticoagulation strategies and indications in the very elderly. Am J Geriatr Cardiol. 2002;11:357-364.

6. Herzog CA, Asinger RW, Berger AK, et al. Cardiovascular disease in chronic kidney disease: a clinical update from Kidney Disease: Improving Global Outcomes (KDIGO). Kidney Int. 2011;80:572-586.

7. Collins AJ, Foley RN, Chavers B, et al. United States Renal Data System 2011 Annual Data Report: Atlas of chronic kidney disease and endstage renal disease in the United States. Am J Kidney Dis. 2012;59:A7. e1-e420.

8. Weiner DE, Tabatabai S, Tighiouart $\mathrm{H}$, et al. Cardiovascular outcomes and all-cause mortality: exploring the interaction between CKD and cardiovascular disease. Am J Kidney Dis. 2006;48:392-401.

9. Benjamin EJ, Levy D, Vaziri SM, et al. Independent risk factors for atrial fibrillation in a population-based cohort. The Framingham Heart Study. JAMA. 1994;271:840-844.

10. Benjamin EJ, Wolf PA, D'Agostino RB, et al. Impact of atrial fibrillation on the risk of death: the Framingham Heart Study. Circulation. 1998;98:946-952.

11. Go AS, Chertow GM, Fan D, et al. Chronic kidney disease and the risks of death, cardiovascular events, and hospitalization. $N$ Engl $J$ Med. 2004;351:1296-1305.

12. Wolf PA, Mitchell JB, Baker CS, et al. Impact of atrial fibrillation on mortality, stroke, and medical costs. Arch Intern Med. 1998;158: 229-234.

13. Bansal N, Fan D, Hsu CY, et al. Incident atrial fibrillation and risk of endstage renal disease in adults with chronic kidney disease. Circulation. 2013;127:569-574.

14. Winkelmayer WC, Patrick AR, Liu J, et al. The increasing prevalence of atrial fibrillation among hemodialysis patients. J Am Soc Nephrol. 2011;22:349-357.

15. Atar I, Konas D, Acikel S, et al. Frequency of atrial fibrillation and factors related to its development in dialysis patients. Int $J$ Cardiol. 2006;106:47-51.

16. Genovesi S, Pogliani D, Faini A, et al. Prevalence of atrial fibrillation and associated factors in a population of long-term hemodialysis patients. Am J Kidney Dis. 2005;46:897-902.

17. Alonso A, Lopez FL, Matsushita K, et al. Chronic kidney disease is associated with the incidence of atrial fibrillation: the Atherosclerosis Risk in Communities (ARIC) study. Circulation. 2011;123:2946-2953.
18. Baber U, Howard VJ, Halperin JL, et al. Association of chronic kidney disease with atrial fibrillation among adults in the United States: Reasons for Geographic and Racial Differences in Stroke (REGARDS) Study. Circ Arrhythm Electrophysiol. 2011;4:26-32.

19. Ananthapanyasut W, Napan S, Rudolph EH, et al. Prevalence of atrial fibrillation and its predictors in nondialysis patients with chronic kidney disease. Clin J Am Soc Nephrol. 2010;5:173-181.

20. Soliman EZ, Prineas RJ, Go AS, et al. Chronic kidney disease and prevalent atrial fibrillation: the Chronic Renal Insufficiency Cohort (CRIC). Am Heart J. 2010;159:1102-1107.

21. Horio T, Iwashima Y, Kamide K, et al. Chronic kidney disease as an independent risk factor for new-onset atrial fibrillation in hypertensive patients. J Hypertens. 2010;28:1738-1744.

22. Genovesi S, Vincenti A, Rossi E, et al. Atrial fibrillation and morbidity and mortality in a cohort of long-term hemodialysis patients. Am J Kidney Dis. 2008;51:255-262.

23. Nakagawa K, Hirai T, Takashima S, et al. Chronic kidney disease and CHADS(2) score independently predict cardiovascular events and mortality in patients with nonvalvular atrial fibrillation. Am J Cardiol. 2011;107:912-916.

24. Anderson JL, Adams CD, Antman EM, et al. ACC/AHA 2007 guidelines for the management of patients with unstable angina/nonST-Elevation myocardial infarction: a report of the American College of Cardiology/American Heart Association Task Force on Practice Guidelines developed in collaboration with the American College of Emergency Physicians, the Society for Cardiovascular Angiography and Interventions, and the Society of Thoracic Surgeons endorsed by the American Association of Cardiovascular and Pulmonary Rehabilitation and the Society for Academic Emergency Medicine. JAm Coll Cardiol. 2007;50:e1-e157.

25. Thygesen K, Alpert JS, White HD, et al. Joint ESC/ACCF/AHA/WHF Task Force for the Redefinition of Myocardial Infarction. Universal definition of myocardial infarction. Circulation. 2007;116:2634-2653.

26. Fox K, Garcia MA, Ardissino D, et al. Guidelines on the management of stable angina pectoris: executive summary: The Task Force on the Management of Stable Angina Pectoris of the European Society of Cardiology. Eur Heart J. 2006;27:1341-1381.

27. Schiller NB, Shah PM, Crawford M, et al. Recommendations for quantitation of the left ventricle by two-dimensional echocardiography. American Society of Echocardiography Committee on Standards, Subcommittee on Quantitation of Two-Dimensional echocardiograms. J Am Soc Echocardiogr. 1989;2:358-367.

28. Devereux RB, Alonso DR, Lutas EM, et al. Echocardiographic assessment of left ventricular hypertrophy: comparison to necropsy findings. Am J Cardiol. 1986;57:450-458.

29. Fuster V, Rydén LE, Cannom DS, et al. ACC/AHA/ESC 2006 Guidelines for the Management of Patients with Atrial Fibrillation: a report of the American College of Cardiology/American Heart Association Task Force on Practice Guidelines and the European Society of Cardiology Committee for Practice Guidelines (Writing Committee to Revise the 2001 Guidelines for the Management of Patients With Atrial Fibrillation): developed in collaboration with the European Heart Rhythm Association and the Heart Rhythm Society. Circulation. 2006;114:e257-e354.

30. Dickstein K, Cohen-Solal A, Filippatos G, et al. ESC Guidelines for the diagnosis and treatment of acute and chronic heart failure 2008: the Task Force for the Diagnosis and Treatment of Acute and Chronic Heart Failure 2008 of the European Society of Cardiology. Developed in collaboration with the Heart Failure Association of the ESC (HFA) and endorsed by the European Society of Intensive Care Medicine (ESICM). Eur Heart J. 2008;29:2388-2442.

31. Ma YC, Zuo L, Chen JH, et al. Modified glomerular filtration rate estimating equation for Chinese patients with chronic kidney disease. J Am Soc Nephrol. 2006;17:2937-2944.

32. National Kidney Foundation. K/DOQI clinical practice guidelines for chronic kidney disease: evaluation, classification, and stratification. $\mathrm{Am}$ J Kidney Dis. 2002;39:S1-S266. 
33. Schlaich MP, Socratous F, Hennebry S, et al. Sympathetic activation in chronic renal failure. J Am Soc Nephrol. 2009;20:933-939.

34. Ehrlich JR, Hohnloser SH, Nattel S. Role of angiotensin system and effects of its inhibition in atrial fibrillation: clinical and experimental evidence. Eur Heart J. 2006;27:512-518.

35. Ott A, Breteler MM, de Bruyne MC, et al. Atrial fibrillation and dementia in a population-based study. The Rotterdam Study. Stroke. $1997 ; 28: 316-321$
36. Hart RG, Pearce LA, Asinger RW, et al. Warfarin in atrial fibrillation patients with moderate chronic kidney disease. Clin J Am Soc Nephrol. 2011;6:2599-2604.

37. Nelson SE, Shroff GR, Li S, et al. Impact of chronic kidney disease on risk of encident atrial fibrillation and subsequent survival in medicare patients. J Am Heart Assoc. 2012;1:e02097.

\section{Publish your work in this journal}

Clinical Interventions in Aging is an international, peer-reviewed journal focusing on evidence-based reports on the value or lack thereof of treatments intended to prevent or delay the onset of maladaptive correlates of aging in human beings. This journal is indexed on PubMed Central, MedLine, the American Chemical Society's 'Chemical Abstracts Ser- vice' (CAS), Scopus and the Elsevier Bibliographic databases. The manuscript management system is completely online and includes a very quick and fair peer-review system, which is all easy to use. Visit $\mathrm{http}: / / \mathrm{www}$.dovepress.com/testimonials.php to read real quotes from published authors.

Submit your manuscript here: http://www.dovepress.com/clinical-interventions-in-aging-journal 